\title{
Non-linear Optical Response of Phenoxazine-based Dyes: Molecular Engineering of Thiadiazole Derivatives as $\pi$-spacers
}

\author{
Muhammad Ramzan Saeed Ashraf Janjua ${ }^{1 *}$ \\ 1 Department of Chemistry, King Fahd University of Petroleum \& Minerals (KFUPM) Dhahran 31261, \\ Kingdom of Saudi Arabia \\ * To whom correspondence should be addressed. E-mail: Janjua@kfupm.edu.sa / Janjua@uos.edu.pk \\ Phone: +966-550442019 / +92-300-6604948
}

TITLE RUNNING HEAD: NLO response of phenoxazine-based dyes

Received May $2^{\text {nd }}, 2017$; Accepted May $16^{\text {th }}, 2017$.

\begin{abstract}
In this article, various dyes have been quantum chemically designed which are based on A- $\pi-\mathrm{D}-\pi-\mathrm{A}$ structural configuration. The main objective of this work is to study the effect of electron deficient thiadiazole derivatives as the $\pi$-spacers. The role of these $\pi$-spacers in phenoxazine-based dyes have been studied through electronic, non-linear optical (NLO) and absorption properties. The electronic structures, absorption spectra and NLO response were calculated by employing quantum chemical methods. The results reveal that the polarizability $(\alpha)$ and hyperpolarizability $(\beta)$ remarkably increase from dye $\mathbf{1}$ to dye 5. As compared to model of dye $\mathbf{1}$, dyes $\mathbf{2 - 5}$ have shown red-shifted absorption spectra and low band gaps.
\end{abstract}

Keywords: Organic dyes; Polarizability; Hyperpolarizability; Phenoxazine; DFT.

\section{Introduction}

An easy, low cost synthesis and fabrication of organic compounds make them suitable for electronic applications. Structural versatility of organic compounds allow easy tuning of the chemical structures and properties.[1] It is well-known that the intramolecular charge transfer (CT) strongly affects first hyperpolarizabilities/second order non-linear optical properties.[2]

To get higher efficiency, a dye should have higher molar extinction coefficients in visible and IR region. The selection of conjugating length is very important to control molar extinction coefficients and light harvesting ability. [3]

Organic molecule acceptors mostly display narrow absorption band in visible region. One of the effective method to lower the highest occupied molecular orbital (HOMO)-the lowest unoccupied molecular orbital (LUMO) gap is by lifting the energy of HOMO while at the same time maintaining the suitable LUMO energy level.

Generally, electron-deficient units like 2,1,3-benzothiadiazole (BT) and thiadiazolo[3,4-c]pyridine (PyT) are used to lower LUMO level of molecules, same time to maintain the HOMO level which may lead to lower HOMO-LUMO band
Resumen. En este trabajo se utilizó la química cuántica para el diseño de varios pigmentos basados en configuraciones estructurales A- $\pi$-D$\pi$-A. El objetivo fundamental del trabajo fue estudiar el efecto de derivados tiadiazol deficientes de electrones como espaciadores $\pi$. El papel de estos espaciadores $\pi$ en pigmentos basados en fenoxazin fue estudiado mediante óptica no linear (ONL) electrónica y propiedades de absorción. Las estructuras electrónicas, los espectros de absorción y la respuesta ONL se calcularon empleando métodos de la química cuántica. Los resultados revelan que la polarizabilidad $(\alpha)$ y la hiperpolarizabilidad $(\beta)$ se incrementan marcadamente del pigmento 1 al 5 . Comparados con el pigmento 1, los pigmentos del 2 al 5 mostraron espectros de absorción con desplazamiento al rojo y pequeños anchos de banda.

Palabras clave: pigmentos orgánicos; polarizabilidad; hiperpolarizabilidad; fenoxazin, TFD.

gap. This strategy provides a red-shifted UV/Vis spectrum [34]. To further decrease the HOMO-LUMO energy gap, a naphtho[2,1-b:3,4-b']dithiophene (NDT) unit is connected to BT or PyT to lift the HOMO energy levels. The structures of the dyes studied in this work are shown in Fig. 1 where phenoxazine moiety is used as electron-donating moiety while cyanoacrylic acid as an electron acceptor group. The electron-deficient units BT (dye 2), PyT (dye 3), NDT-BT (dye 4), and NDT-PyT (dye 5 ) are involved as $\pi$-conjugating bridges between donor-acceptor systems. The new phenoxazine-based dyes have been denoted as dye 2 to dye 5 . A model of dye 1 with phenylene as the $\pi$-spacer was also studied for parallel comparison. In dye 2 and dye 3 , the only structural difference is that dye 2 contains a BT unit while dye 3 is equipped with a PyT unit. Computational chemistry methods are used to calculate the electronic, optical and non-linear optical properties. We do hope that this work may help to synthesize efficient organic compounds in future.

\section{Computational details}

All the calculations were performed using Gaussian 09 program package [5]. Geometry optimization was carried out by 
B3LYP (Becke, 3-parameter, Lee-Yang-Parr) functional and 6-31G $(\mathrm{d}, \mathrm{p})$ basis set [5]. The lack of imaginary values in the wave numbers calculations indicated the successful geometry optimization. A tight SCF convergence criteria $\left(10^{-8}\right.$ a.u. $)$ and integration grid of $10^{-8}$ were used for all calculations. DFT and TD-DFT are considered as good methodologies to calculate optical properties and have already shown good results [6]. The long-range correction of B3LYP, Coulomb-attenuating method (CAM-B3LYP) was used to calculate absorption spectra of designed dyes. The success of this methodology is already well-established for organic dyes [7]. TD-DFT and solvent effect (methanol) was undertaken using conductor-like polarizable continuum model (CPCM). It has been mentioned in revised manuscript in computational part [8]. During these calculations the 20 lowest singlet-singlet transitions were studied.

Average polarizability $(\alpha)$ is determined using following formula:[8]

$$
\alpha=1 / 3\left(\alpha_{x x}+\alpha_{y y}+\alpha_{z z}\right)
$$

Whereas the ten components $\left(\beta_{\mathrm{xxx}}, \beta_{\mathrm{xxy}}, \beta_{\mathrm{xyy}}, \beta_{\mathrm{yyy}}, \beta_{\mathrm{xxz}}\right.$, $\beta_{\mathrm{xyz}}, \beta_{\mathrm{yyz}}, \beta_{\mathrm{xzz}}, \beta_{\mathrm{yzz}}$ and $\beta_{\mathrm{zzz}}$ ) present in Gaussian $09 \mathrm{~W}$ output file have been considered to calculate first hyperpolarizability $\left(\beta_{\text {tot }}\right)$ by employing following equation:[9]

$$
\begin{aligned}
\beta_{t o t} & =\left[\left(\beta_{x x x}+\beta_{x y y}+\beta_{x z z}\right)^{2}+\left(\beta_{y y y}+\beta_{x x y}+\beta_{y z z}\right)^{2}\right. \\
& \left.+\left(\beta_{z z z}+\beta_{x x z}+\beta_{y y z}\right)^{2}\right]^{1 / 2}
\end{aligned}
$$

\section{Results and discussion}

In this article, we have presented the results of theoretical designing of NLO dyes. The different dyes with A- $\pi$-D- $\pi$-A configuration have been quantum mechanically designed. The computational orientation and structures of dyes are given in Fig. 1.

DFT and TDDFT calculations were performed to shed light on how one donor moiety influence the NLO properties within two acceptors. Using theoretical methods, we have calculated following properties. (i) polarizability $(\alpha)$ (ii) hyperpolarizability $(\beta)$ and (iii) absorption wave length.

\subsection{HOMO-LUMO energy band gap}

To understand the electronic and spectroscopic properties molecules, it is important to have knowledge of the molecular geometries and electron density distribution of the organic dyes. From table 1. It is clear that energy levels of designed dyes are very sensitive to length of conjugated system. The electron-deficient thiadiazole derivatives in conjugated systems lower the LUMO level and decrease the band gap.

Table 1. HOMO, LUMO and band gap of designed systems $(\mathrm{eV})$.

\begin{tabular}{cccc}
\hline Dye & HOMO & LUMO & Band gap \\
\hline 1 & -5.463 & -3.007 & 2.455 \\
2 & -5.597 & -3.889 & 1.708 \\
3 & -5.779 & -4.203 & 1.576 \\
4 & -5.244 & -3.749 & 1.495 \\
5 & -5.293 & -4.032 & 1.261 \\
\hline
\end{tabular}

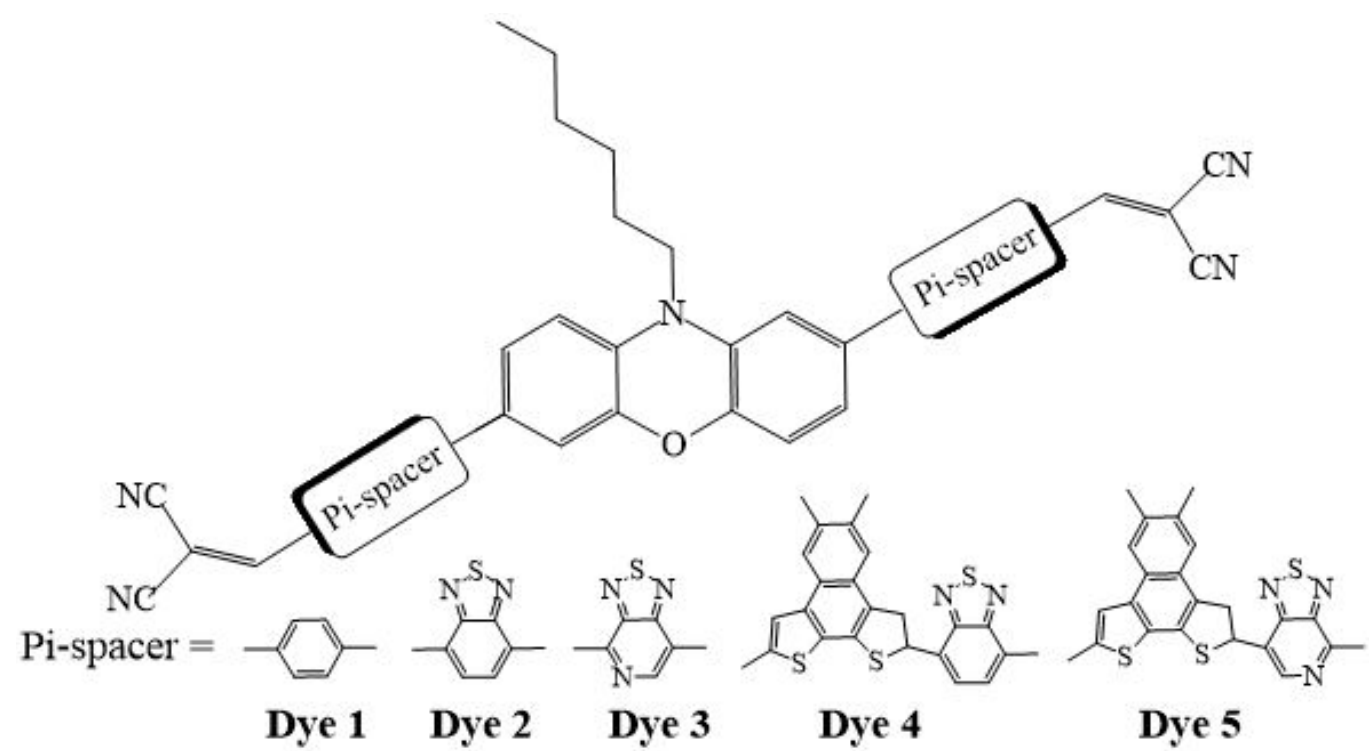

Fig. 1. Orientation and structures of designed systems. 
BT is considered an excellent group to build low band gap dyes because it significantly lowers the LUMO energy level. As a result red-shifted adsorption spectrum can be obtained. In dye 2, use of electron-deficient BT unit lower the LUMO to -3.889 as compared with dye 1 which contains benzene/phenylene $(-3.007 \mathrm{eV})$. While HOMO levels of Dye 1 and Dye 2 are almost similar. Band gap of Dye2 is $1.708 \mathrm{eV}$ lower than Dye 1. Similar trend has also been observed for Dye 3 and Dye 1 . However, a pyridine unit is more $\pi$-electron deficient, replacement of benzene with pyridine has given a new unit thiadiazolo[3,4-c]pyridine (PyT). Structural comparison between dyes 2-5 indicates that the replacement of BT with PyT significantly lowers the LUMO energy level as shown in table 1. On comparing dyes 1-4, it has been observed that the replacement of electron-deficient BT unit with more electron-deficient PyT unit effectively lowers the LUMO energy and therefore decreases the band gap. Thiophene is an electron-rich, it increases the HOMO energy level, thus decreases the band gap. One can also decrease the electron-richness of thiophene unit by fusing it with a less electron-rich benzene unit. On comparing dye 3 and dye 4 , the incorporation of NDT unit into the $\pi$-linkage raises the HOMO energy level of dye 4 to $-5.244 \mathrm{eV}$ as compared with that of dye $3(-5.779 \mathrm{eV})$.

\subsection{Non-linear optical properties}

Non-linear optical materials are used in communication technology, optical switches and signal processing. So, study of non-linear optical properties are very important. Optical response depends upon on the polarizability ( $\alpha$, linear response) and hyperpolarizabilities ( $\beta, \gamma$, etc. nonlinear responses).
Table 2. Dipole polarizabilities and major contributing tensors (a.u)

\begin{tabular}{ccccc}
\hline Dye & $\alpha_{\mathrm{XX}}$ & $\alpha_{\mathrm{YY}}$ & $\alpha_{\mathrm{ZZ}}$ & $\alpha$ \\
\hline 1 & 1124.74 & 329.31 & 174.09 & 582.81 \\
2 & 1601.19 & 482.31 & 157.54 & 800.64 \\
3 & 1900.97 & 489.55 & 141.39 & 1322.90 \\
4 & 2631.94 & 1758.1 & 311.17 & 1490.20 \\
5 & 2847.03 & 1838.6 & 329.74 & 1671.79 \\
\hline
\end{tabular}

Calculation of these properties is very important to assess the non-linear optical potential of molecules. Values of average polarizability and major contributing tensors of five dyes are given in Table 2. Order of average polarizability of designed systems was: Dye $5>$ Dye $4>$ Dye $3>$ Dye $2>$ Dye 1 . As we discuss in above section, introduction of electron deficient group in pi-spacer remarkably decrease the band gap. As a result, polarity significantly increases. Dye 2 with BT group shows polarizability of 800 a.u, which is higher than Dye 1, because BT group decrease the band gap by lowering the LUMO level. Dye 3 has low band gap as compared to dyes 1 and 2 and shows higher polarizability (1322 a.u).

Comparison among Dye 2 and Dye 3, Dye 4 and Dye 5 indicate that the replacement of BT with PyT significantly increase the polarizability. Basically, pyridine unit is more $\pi$-electron deficient than benzene. So, PyT is more electron-deficient and responsible for lower band gap. Generally, a large linear polarizability is required to obtain large hyperpolarizabilities. [11] Hyperpolarizabilities were calculated by using B3LYP functional. The computed $\beta_{\text {tot }}$ values and major contributing

Table 3. First hyperpolarizabilities $\beta$ tot and major contributing tensors (a.u)

\begin{tabular}{|c|c|c|c|c|c|c|}
\hline Dyes & $\beta_{X X X}$ & $\beta_{X X Y}$ & $\beta_{Y Y Y}$ & $\beta_{X Y Z}$ & $\beta_{Y Y Z}$ & $\beta_{\text {tot }}$ \\
\hline 1 & $\begin{array}{c}12652.69 \\
(12401.11)\end{array}$ & $\begin{array}{c}-3639.10 \\
(-3489.62)\end{array}$ & $\begin{array}{c}-51.78 \\
(-46.83)\end{array}$ & $\begin{array}{c}-134.22 \\
(129.92)\end{array}$ & $\begin{array}{c}26.00 \\
(23.96)\end{array}$ & $\begin{array}{c}13660.62 \\
(13432.12)\end{array}$ \\
\hline 3 & -25990.80 & 6983.87 & 187.28 & -506.15 & -65.94 & 20283.83 \\
\hline 4 & 22945.03 & 150508.80 & 51416.04 & 6691.45 & -1015.47 & 247225.4 \\
\hline
\end{tabular}

(The values given in parentheses are obtained by employing diffused function $6-31+\mathrm{G}^{*}$ )

Table 4. Computed maximum absorption wavelengths $\left(\lambda_{\max } / \mathrm{nm}\right)$, Transition energy $\mathrm{E}_{\mathrm{gm}}(\mathrm{eV})$, oscillator strengths $(f)$, transition moment $\left(\mathrm{M}_{x}^{g e}\right.$ a.u.) and transition natures of dyes (1-5).

\begin{tabular}{cccccc}
\hline Acceptor & $\lambda_{\max }$ & $\mathrm{E}_{\mathrm{g}}$ & $\mathrm{f}$ & $\mathrm{M}_{\mathrm{x}}{ }_{\mathrm{ge}}$ & Main configurations \\
\hline 1 & 375 & 3.299 & 1.062 & 1.88 & HOMO $\rightarrow$ LUMO $(69 \%)$ \\
2 & 547 & 2.262 & 0.867 & 1.91 & HOMO $\rightarrow$ LUMO $(70 \%)$ \\
3 & 615 & 2.012 & 0.988 & 1.94 & HOMO $\rightarrow$ LUMO $(84 \%)$ \\
4 & 581 & 2.129 & 1.245 & 1.97 & HOMO $\rightarrow$ LUMO $(63 \%)$ \\
5 & 639 & 1.936 & 1.019 & 1.98 & HOMO $\rightarrow$ LUMO $(45 \%)$ \\
\hline
\end{tabular}


tensor of systems are given in Table 3. Order $\beta_{\text {tot }}$ of five dyes is: Dye $5>$ Dye $4>$ Dye $3>$ Dye $2>$ Dye 1 . Higher NLO response may be due to enhance charge transfer from donor to acceptor through $\pi$-spacer along $\mathrm{x}$-axis.

\subsection{UV-Vis spectra and HOMO-LUMO of dyes}

DFT/TDDFT computational methodologies are considered to be reliable for organic dyes [7-11]. The CAM-B3LYP functional has been reported to provide reliable results for some organic dyes, with excitation energies very close to experimental data [11].

As compared to Dye 1 with phenylene as $\pi$-spacer, the introduction of BT, PyT, NDT-BT, and NDT-PyT moieties have strong impact on UV/Vis spectra and it has led to remarkably red-shifted adsorption bands. Dye 2 with BT unit has shown a remarkable red-shifted absorption by $72 \mathrm{~nm}$ than Dye 1 . BT has strongly affected the electronic structure of dyes by lowering the LUMO level and responsible for red-shift [3]. PyT is more electron-deficient group than BT, so it has shown more red-shifted absorption than Dye1. Dye 2 and Dye 3 with almost similar structure, Dye 3 has shown a $68 \mathrm{~nm}$ red-shifted absorption band as compared to the Dye 2, in fact that the pyridine unit is a more stronger $\pi$-electron deficient unit.

From table 4, it can been seen that Dye 4 has shown absorbance maxima at $581 \mathrm{~nm}$ that's $206 \mathrm{~nm}$ red-shifted than Dye 1 . Dye 5 has most electron-deficient $\pi$-spacer shown a significant increase in red-shift by $264 \mathrm{~nm}$ as compared to Dye 1 . The wide absorption bands over whole visible and near infrared are considered to be a good strategy to enhance photocurrent response. We have increased the absorption wavelength from 375 (dye 1) to 639 (dye 5) nm by structural modification.

The TDDFT results show that the electron transition of dye 1 mainly arises from phenoxazine (HOMO) to left cyanoacrylic acid (LUMO) along the $\mathrm{x}$-direction and this character of charge transfer similarly occurs on dyes 2-5 (HOMO to LUMO). HOMO to LUMO transition is most probable and low energy transition. The HOMOs and LUMOs are shown in Fig. 3. As can be seen from the figure 3 , both the highest occupied molecular orbitals and the lowest-lying unoccupied molecular orbitals are delocalized over phenoxazine and left cyano group respectively due to higher value of $\beta_{X X X}$ component in all the studied dyes (1-5). However, the direction of charge transfer is from phenoxazine donor towards cyanoacrylic acid acceptor group.

\subsection{First hyperpolarizability}

In order to have good understanding about First hyperpolarizability or NLO response $(\beta)$, the information of dipole polarizability is also important. The average polarizability, $\langle\alpha\rangle$ is defined as:

$$
<\alpha>=1 / 3\left(\alpha_{x x}+\alpha_{y y}+\alpha_{z z}\right)
$$

There are three components of dipole polarizability $\alpha_{x x}, \alpha_{y y}$ and $\alpha_{z z}$ respectively. There is $\alpha_{x x}$ component has the largest value as compared to the $\alpha_{z z}$ and $\alpha_{y y}$ components and the property of the studied dyes (1-5) is mainly assessed by the $x$-direction transition (Table 1 and Fig. 1). The mathematical formulation of dipole polarizability along $x$-direction is defined in the following equation:

$$
\alpha_{x x} \propto \frac{\left(M_{x}^{g e}\right)^{2}}{E_{g e}}
$$

According to equation (4), we know that the $\alpha$ value is directly proportional to the square of transition moment, and is inversely proportional to the transition energy. As a result, the system with least transition energy will have a larger $\alpha$ value. The excitation energies $(E)$, transition moments $\left(M_{x}^{g e}\right)$, and corresponding dominant molecular orbital transitions of dyes $\mathbf{1} \mathbf{- 5}$ are provided in Table 4 . The computed $M_{x}^{g e}$ values of studied dyes $\mathbf{1}-\mathbf{5}$ are increasing as follows $\mathbf{1}<\mathbf{2}<\mathbf{3}<\mathbf{4}<\mathbf{5}$; the

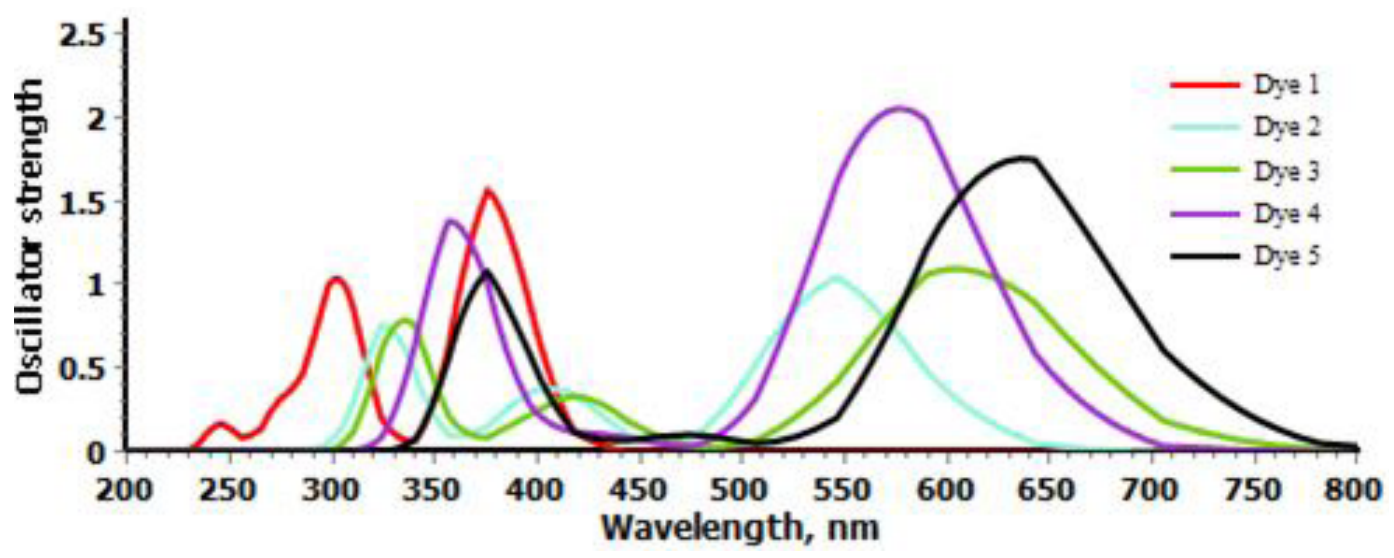

Fig. 2. Simulated Absorption spectra of Dyes. 

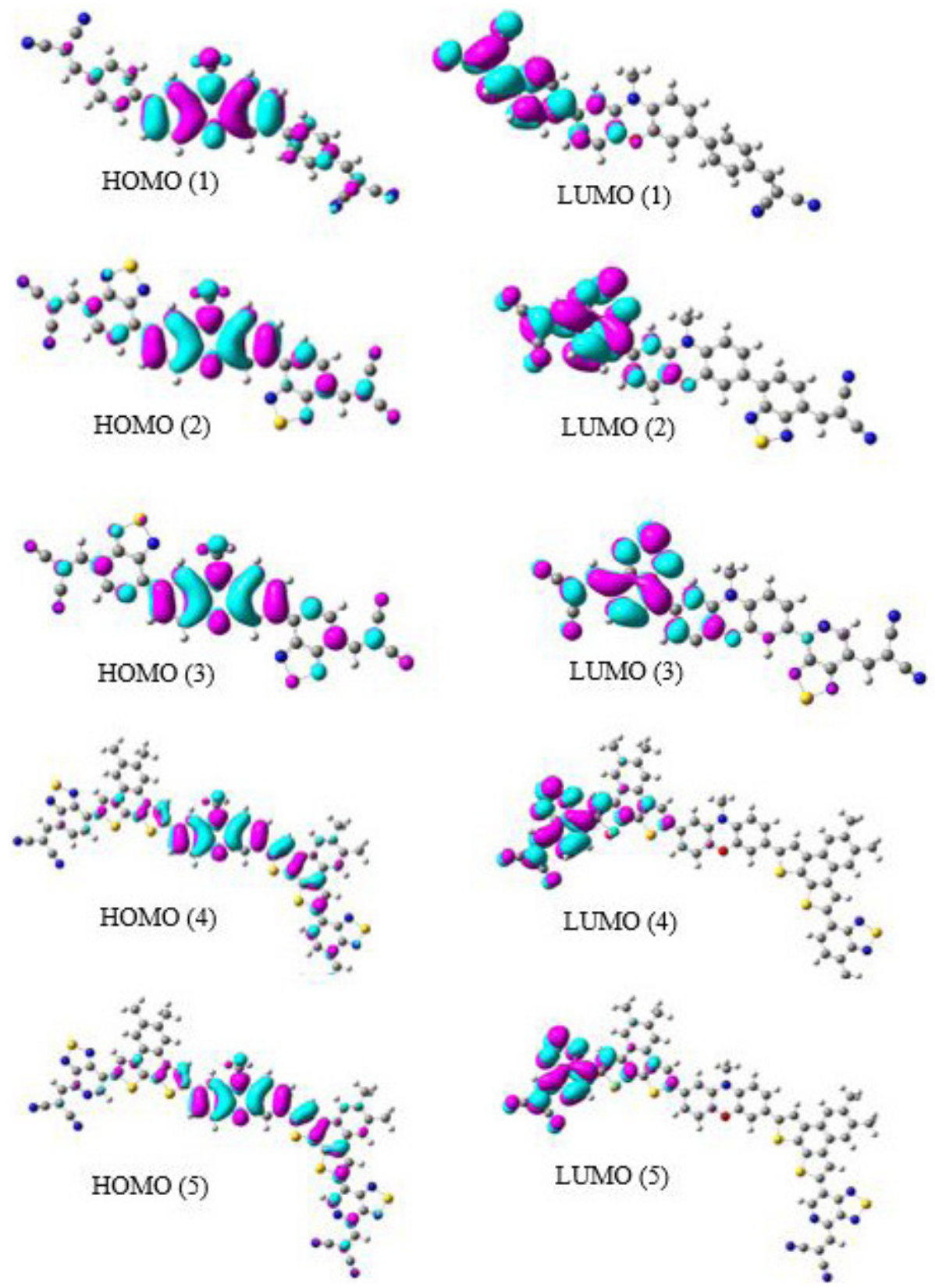

Fig. 3. Frontier Molecular Orbitals of Systems (1-5).

average polarizability, $\langle\alpha\rangle$ is in the following order: system $\mathbf{5}\rangle$ $\mathbf{4}>\mathbf{3}>\mathbf{2}>\mathbf{1}$ as shown in Table 2. The greater the value of $M_{x}^{g e}$ along $\mathrm{x}$-axis means that the greater is the impact of charge transfer in that direction which leads to greater value of hyperpolarizability. Therefore, the order $\beta_{\text {tot }}$ of five dyes is: Dye $\mathbf{5}>$ Dye $\mathbf{4}>$ Dye $\mathbf{3}>$ Dye $\mathbf{2}>$ Dye $\mathbf{1}$ as shown in table 4. Generally, the low-lying HOMO-LUMO energy gap might enhance molecular first hyperpolarizability. The energy gaps of studied dyes are decreasing in the following order: system $\mathbf{1}>\mathbf{2}>\mathbf{3}>$ $\mathbf{4}>\mathbf{5}$ while the NLO response is increasing accordingly as follows: $\mathbf{1}<\mathbf{2}<\mathbf{4}<\mathbf{3}<\mathbf{5}$.

\section{Conclusions}

The DFT and TDDFT methods have been used to explain effect of pi-spacer on NLO and optical properties. The band gap is significantly reduced from 2.455 to $1.261 \mathrm{eV}$ by the modification of $\pi$-spacers. The polarizabilities are increased from 582.81 a.u to 1671.79 a.u from dye 1 to dye 5. In this way, the hyperpolarizabilities have been increased from 13660.62 a.u to 295926.1 a.u in studied dyes. Though, by structural modifications we have achieved significant red shifted absorption spectrum from $375 \mathrm{~nm}$ (dye 1) to $639 \mathrm{~nm}$ (dye 5). We do hope that 
this work might be helpful for the molecular design of organic dyes with targeted properties to improve the performance in opto-electronics.

\section{Acknowledgement}

The author would like to acknowledge the support provided by the Deanship of Scientific Research (DSR) at King Fahd University of Petroleum \& Minerals (KFUPM) for funding this work through project No. SR161009.

\section{References}

1. Williams, D. J. Angewandte Chemie International Edition in English 1984, 23, 690-703.

2. a) J. Zyss, D. S. Chemla, in Nonlinear Optical Properties of Organic Molecules and Crystals (Ed.: D. S. C. Zyss), Academic Press, 1987, pp. 23-191; b) S. P. Karna, Y. Zhang, M. Samoc, P. N. Prasad, B. A. Reinhardt, A. G. Dillard, The Journal of Chemical Physics 1993, 99, 9984-9993; c) M. Drozd, M. K. Marchewka, Journal of Molecular Structure: THEOCHEM 2005, 716, 175192; d) R.-L. Zhong, H.-L. Xu, Z.-R. Li, Z.-M. Su, The Journal of Physical Chemistry Letters 2015, 6, 612-619; e) P. Z.-H. Zeng Yi, Zhao Fu-Li, Qin Mu, Zhou Yan, Wang Chang-Shun, Chinese Phys. B 2014, 23, 024212.f) M.R.S.A. Janjua*, Z. H. Yamani, S. Jamil, Z. Yang, S. Pan, First principle study of electronic and non-linear optical (NLO) properties of triphenylamine dyes: Interactive design computation of new NLO compounds, Aust. J. Chem. 69 (2016) 467-472. g) M.R.S.A. Janjua*, S. Jamil, A. Mahmood, A. Zafar, M. Haroon and H. N. Bhatti, Aust. J. Chem. 68 (2015), 1502-1507. h) M.R.S.A. Janjua*, A. Mahmood, M. F. Nazar, Z.H. Yang, and S.L. Pan, Acta Chim. Sol. 61 (2014), 382-390. i) M.R.S.A. Janjua, Inorg. Chem. 51 (2012), 11306-11314. j) M.R.S.A. Janjua, M. Amin, M. Ali, B. Bashir, M.U. Khan, M.A. Iqbal, W. Guan, L.K. Yan, Z.M. Su, Eur. J. Inorg. Chem. (2012), 705-711 k) M.R.S.A. Janjua, W. Guan, L.K. Yan, Z.M. Su, Curr. Phys. Chem. 1 (2011), 99-105. 1) M.R.S.A. Janjua, W. Guan, L.K. Yan, Z.M. Su, M. Ali, I.H. Bukhari, J. Mol. Graph. Model. 28 (2010), 735-745. m) M.R.S.A. Janjua, Z.M. Su, W. Guan, C.G. Liu, L.K. Yan, P. Song, G. Maheen, Aust. J. Chem. 63 (2010), 836844. n) M.R.S.A. Janjua, C.G. Liu, W. Guan, S. Muhammad, L.K. Yan, Z.M. Su, J. Phys. Chem. A 113 (2009), 3576-3587. o) M.R.S.A. Janjua, W. Guan, C.G. Liu, S. Muhammad, L.K. Yan,
Z.M. Su, Eur. J. Inorg. Chem. (2009), 5181-5188. p) B. Zhang, G. Shi, Z.H. Yang, F.F. Zhang, S.L. Pan, Angew. Chem. Int. Ed., 2017, 56, 3916-3919. q) M. Zhang, X. Su, M. Mutailipu, Z.H. Yang, S.L. Pan, Chem. Mater., 2017, 29, 945-949.

3. a) K. R. Justin Thomas, P. Singh, A. Baheti, Y.-C. Hsu, K.-C. Ho, J. T. s. Lin, Dyes and Pigments 2011, 91, 33-43; b) M. Velusamy, K. R. Justin Thomas, J. T. Lin, Y.-C. Hsu, K.-C. Ho, Organic Letters 2005, 7, 1899-1902.

4. aD. H. Lee, M. J. Lee, H. M. Song, B. J. Song, K. D. Seo, M. Pastore, C. Anselmi, S. Fantacci, F. De Angelis, M. K. Nazeeruddin, M. Gräetzel, H. K. Kim, Dyes and Pigments 2011, 91, 192-198; bY.-S. Yen, Y.-C. Hsu, J. T. Lin, C.-W. Chang, C.-P. Hsu, D.-J. Yin, The Journal of Physical Chemistry C 2008, 112, 12557-12567.

5. M. J. Frisch, G. W. Trucks, H. B. Schlegel, G. E. Scuseria, M. A. Robb, J. R. Cheeseman, G. Scalmani, V. Barone, B. Mennucci, G. A. Petersson, H. Nakatsuji, M. Caricato, X. Li, H. P. Hratchian, A. F. Izmaylov, J. Bloino, G. Zheng, J. L. Sonnenberg, M. Hada, M. Ehara, K. Toyota, R. Fukuda, J. Hasegawa, M. Ishida, T. Nakajima, Y. Honda, O. Kitao, H. Nakai, T. Vreven, J. A. Montgomery Jr., J. E. Peralta, F. Ogliaro, M. J. Bearpark, J. Heyd, E. N. Brothers, K. N. Kudin, V. N. Staroverov, R. Kobayashi, J. Normand, K. Raghavachari, A. P. Rendell, J. C. Burant, S. S. Iyengar, J. Tomasi, M. Cossi, N. Rega, N. J. Millam, M. Klene, J. E. Knox, J. B. Cross, V. Bakken, C. Adamo, J. Jaramillo, R. Gomperts, R. E. Stratmann, O. Yazyev, A. J. Austin, R. Cammi, C. Pomelli, J. W. Ochterski, R. L. Martin, K. Morokuma, V. G. Zakrzewski, G. A. Voth, P. Salvador, J. J. Dannenberg, S. Dapprich, A. D. Daniels, Ö. Farkas, J. B. Foresman, J. V. Ortiz, J. Cioslowski, D. J. Fox, Gaussian, Inc., Wallingford, CT, USA, 2009.

6. aJ. Preat, C. Michaux, D. Jacquemin, E. A. Perpète, The Journal of Physical Chemistry C 2009, 113, 16821-16833; bS. Kim, J. K. Lee, S. O. Kang, J. Ko, J. H. Yum, S. Fantacci, F. De Angelis, D. Di Censo, M. K. Nazeeruddin, M. Grätzel, Journal of the American Chemical Society 2006, 128, 16701-16707; cD. Jacquemin, V. Wathelet, E. A. Perpète, C. Adamo, Journal of Chemical Theory and Computation 2009, 5, 2420-2435; dM. Pastore, E. Mosconi, F. De Angelis, M. Grätzel, The Journal of Physical Chemistry C 2010, 114, 7205-7212.

7. T. Yanai, D. P. Tew, N. C. Handy, Chemical Physics Letters 2004, 393, 51-57.

8. V. Barone, M. Cossi, The Journal of Physical Chemistry A 1998, 102, 1995-2001.

9. A. Karakas, A. Elmali, H. Unver, Spectrochimica Acta Part A: Molecular and Biomolecular Spectroscopy 2007, 68, 567-572.

10. C. Qin, A. E. Clark, Chemical Physics Letters 2007, 438, 26-30.

11. B. A. Sriyanka Mendis, K. M. Nalin de Silva, Journal of Molecular Structure: THEOCHEM 2004, 678, 31-38. 